\title{
The iterative methods for solving nonlinear matrix equation $X+A^{\star} X^{-1} A+B^{\star} X^{-1} B=Q$
}

Sarah Vaezzadeh', Seyyed Mansour Vaezpour ${ }^{1}$, Reza Saadati ${ }^{2}$ and Choonkil Park ${ }^{3 *}$

"Correspondence:

baak@hanyang.ac.kr

${ }^{3}$ Research Institute for Natural

Sciences, Hanyang University, Seoul,

133-791, Korea

Full list of author information is

available at the end of the article

\section{Abstract}

In this paper, we study the matrix equation $X+A^{\star} X^{-1} A+B^{\star} X^{-1} B=Q$, where $A$ and $B$ are square matrices, and $Q$ is a positive definite matrix, and propose the iterative methods for finding positive definite solutions of the matrix equation. Also, general convergence results for the basic fixed point iteration for these equations are given. Some numerical examples are presented to show the usefulness of the iterations.

MSC: 65F10;65F30; 65H10; 15A24

Keywords: nonlinear matrix equation; positive definite solution; inversion-free variant iterative method; convergence rate

\section{Introduction}

In this paper, we consider the matrix equation

$$
X+A^{\star} X^{-1} A+B^{\star} X^{-1} B=Q
$$

where $A$ and $B$ are square matrices, $Q$ is a positive definite matrix. It is easy to see that matrix equation (1) can be reduced to

$$
X+A^{\star} X^{-1} A+B^{\star} X^{-1} B=I,
$$

where $I$ is the identity matrix. Trying to solve special linear systems [1] leads to solving nonlinear matrix equations of the above types as follows.

For a linear system $M x=f$ with

$$
M=\left(\begin{array}{ccc}
Q & 0 & A \\
0 & Q & B \\
A^{\star} & B^{\star} & Q
\end{array}\right)
$$

positive definite, we rewrite $M=\tilde{M}+K$, where

$$
\tilde{M}=\left(\begin{array}{ccc}
X & 0 & A \\
0 & X & B \\
A^{\star} & B^{\star} & Q
\end{array}\right), \quad K=\left(\begin{array}{ccc}
Q-X & 0 & 0 \\
0 & Q-X & 0 \\
0 & 0 & 0
\end{array}\right)
$$

C 2013 Vaezzadeh et al.; licensee Springer. This is an Open Access article distributed under the terms of the Creative Commons Attribution License (http://creativecommons.org/licenses/by/2.0), which permits unrestricted use, distribution, and reproduction in any medium, provided the original work is properly cited. 
Moreover, we decompose $\tilde{M}$ to the $L U$ decomposition

$$
\tilde{M}=\left(\begin{array}{ccc}
X & 0 & A \\
0 & X & B \\
A^{\star} & B^{\star} & Q
\end{array}\right)=\left(\begin{array}{ccc}
I & 0 & 0 \\
0 & I & 0 \\
A^{\star} X^{-1} & B^{\star} X^{-1} & I
\end{array}\right)\left(\begin{array}{ccc}
X & 0 & A \\
0 & X & B \\
0 & 0 & X
\end{array}\right) .
$$

A decomposition of $\tilde{M}$ exists if and only if $X$ is a positive definite solution of matrix equation (1). Solving the linear system $\tilde{M} y=f$ is equivalent to solving two linear systems with a lower and upper block triangular system matrix. To compute the solution of $M x=f$ from $y$, the Woodbury formula [2] can be applied.

The matrix equation $X+A^{\star} X^{-1} A=Q$ has been studied extensively by many authors [314]. Several conditions for the existence of positive definite solutions and some iterations to find maximal positive definite solutions for these equations were discussed. Apparently, matrix equation (1) generalizes the matrix equation $X+A^{\star} X^{-1} A=Q$.

Matrix equation (2) was studied in [15], and based on some conditions, the authors proved that matrix equation (2) has positive definite solutions. They also proposed two iterative methods to find the Hermitian positive definite solutions of matrix equation (2). They did not analyze the convergence rate of proposed algorithms.

In this paper, we propose two algorithms. We will show that Algorithm (7) is more accurate than Algorithm (3) pointed out in [15]. Also, Algorithm (10) needs less operation in comparison with Algorithm (3). The following notations are used throughout the rest of the paper. The notation $A \geq 0(A>0)$ means that $A$ is Hermitian positive semidefinite (positive definite). For Hermitian matrices $A$ and $B$, we write $A \geq B(A>B)$ if $A-B \geq 0$ $(>0)$. Similarly, by $\lambda_{1}(A)$ and $\lambda_{n}$ we denote, respectively, the maximal and the minimal eigenvalues of $A$. The norm used in this paper is the spectral norm of the matrix $A$, i.e., $\|A\|=\left(\lambda_{1}\left(A^{\star} A\right)\right)^{\frac{1}{2}}$.

\section{Fixed point theorems}

Lemma 1 [8] If $C$ and $P$ are Hermitian matrices of the same order with $P>0$, then $C P C+$ $P^{-1} \geq 2 C$.

In [15] an algorithm that avoids matrix inversion for every iteration, called inversionfree variant of the basic fixed point iteration, and a theorem to find a Hermitian positive definite solution of matrix equation (2) were proposed as follows.

\section{Algorithm 1 [15] Let}

$$
\left\{\begin{array}{l}
X_{0}=Y_{0}=I \\
X_{n+1}=I-A^{\star} Y_{n} A-B^{\star} Y_{n} B, \\
Y_{n+1}=2 Y_{n}-Y_{n} X_{n} Y_{n}, \quad n=0,1,2, \ldots
\end{array}\right.
$$

Theorem 1 [15] Assume that matrix equation (2) has a positive definite solution. Then Algorithm (3) defines a monotonically decreasing matrix sequence $\left\{X_{n}\right\}$ converging to the positive definite matrix $X$ which is a solution of matrix equation (2). Also, the sequence $\left\{Y_{n}\right\}$ defined in Algorithm (3) defines a monotonically increasing sequence converging to $X^{-1}$. 
Although it is not mentioned in the previous theorem that the sequence $\left\{X_{n}\right\}$ converges to the maximal Hermitian positive definite solution of equation (2), during the proof of the theorem in [15], it is obvious. So, $X=X_{\infty}$, where $X_{\infty}$ is the maximal positive definite solution of matrix equation (2) in Theorem 1.

The problem of convergence rate for Algorithm (3) was not considered in [15]. We now establish the following result.

Theorem 2 If matrix equation (2) has a positive definite solution, for Algorithm (1) and any $\epsilon>0$, we have

$$
\left\|Y_{n+1}-X_{\infty}^{-1}\right\| \leq\left(\left\|A X_{\infty}^{-1}\right\|+\left\|B X_{\infty}^{-1}\right\|+\epsilon\right)^{2}\left\|Y_{n-1}-X_{\infty}^{-1}\right\|
$$

and

$$
\left\|X_{n+1}-X_{\infty}\right\| \leq\left(\|A\|^{2}+\|B\|^{2}\right)\left\|Y_{n}-X_{\infty}^{-1}\right\|
$$

for all $n$ large enough.

Proof From Algorithm (3), we have

$$
\begin{aligned}
Y_{n+1} & =2 Y_{n}-Y_{n}\left(I-A^{\star} Y_{n-1} A-B^{\star} Y_{n-1} B\right) Y_{n} \\
& =2 Y_{n}-Y_{n}^{2}+Y_{n} A^{\star}\left(X_{\infty}^{-1}+Y_{n-1}-X_{\infty}^{-1}\right) A Y_{n}+Y_{n} B^{\star}\left(X_{\infty}^{-1}+Y_{n-1}-X_{\infty}^{-1}\right) B Y_{n} \\
& =2 Y_{n}-Y_{n} X_{\infty} Y_{n}+Y_{n} A^{\star}\left(Y_{n-1}-X_{\infty}^{-1}\right) A Y_{n}+Y_{n} B^{\star}\left(Y_{n-1}-X_{\infty}^{-1}\right) B Y_{n} .
\end{aligned}
$$

Thus

$$
\begin{aligned}
X_{\infty}^{-1}-Y_{n+1} & =X_{\infty}^{-1}-Y_{n}+Y_{n} X_{\infty} Y_{n}-Y_{n}+Y_{n} A^{\star}\left(X_{\infty}^{-1}-Y_{n-1}\right) A Y_{n}+Y_{n} B^{\star}\left(X_{\infty}^{-1}-Y_{n-1}\right) B Y_{n} \\
& =\left(X_{\infty}^{-1}-Y_{n}\right) X_{+}\left(X_{\infty}^{-1}-Y_{n}\right)+Y_{n} A^{\star}\left(X_{\infty}^{-1}-Y_{n-1}\right) A Y_{n}+Y_{n} B^{\star}\left(X_{\infty}^{-1}-Y_{n-1}\right) B Y_{n} .
\end{aligned}
$$

Now, since $\left\|Y_{n}-X_{\infty}^{-1}\right\| \leq\left\|Y_{n-1}-X_{\infty}^{-1}\right\|$ and $\lim Y_{n}=X_{\infty}^{-1}$, inequality (4) follows. Also, inequality (5) is true since

$$
X_{n+1}-X_{\infty}=A^{\star}\left(X_{\infty}^{-1}-Y_{n}\right) A+B^{\star}\left(X_{\infty}^{-1}-Y_{n}\right) B
$$

This completes the proof.

The above proof shows that Algorithm (3) should be modified as follows to improve the preceding convergence properties.

\section{Algorithm 2 Let}

$$
\left\{\begin{array}{l}
X_{0}=Y_{0}=I, \\
Y_{n+1}=2 Y_{n}-Y_{n} X_{n} Y_{n}, \\
X_{n+1}=I-A^{\star} Y_{n+1} A-B^{\star} Y_{n+1} B, \quad n=0,1,2, \ldots
\end{array}\right.
$$

Theorem 3 Assume that matrix equation (2) has a positive definite solution. Then Algorithm (7) defines a monotonically decreasing matrix sequence $\left\{X_{n}\right\}$ converging to $X_{\infty}$ which 
is the maximal Hermitian positive definite solution of equation (2). Also, the sequence $\left\{Y_{n}\right\}$ defined in Algorithm (7) defines a monotonically increasing sequence converging to $X_{\infty}^{-1}$.

Proof Let $X_{+}$be a positive definite solution of matrix equation (2). It is clear that

$$
X_{0} \geq X_{1} \geq \cdots \geq X_{n} \geq X_{+}, \quad Y_{0} \leq Y_{1} \leq \cdots \leq Y_{n} \leq X_{+}^{-1}
$$

is true for $n=1$. Assume (8) is true for $n=k$. By Lemma 1 , we have that

$$
Y_{k+1}=2 Y_{k}-Y_{k} X_{k} Y_{k} \leq X_{K}^{-1} \leq X_{+}^{-1}
$$

Therefore,

$$
X_{k+1}=I-A^{\star} Y_{k+1} A \geq I-A^{\star} X_{+}^{-1} A=X_{+} .
$$

Since $Y_{k} \leq X_{k-1}^{-1} \leq X_{k}^{-1}$, we have $Y_{k}^{-1} \geq X_{k}$. Thus

$$
Y_{k+1}-Y_{k}=Y_{k}\left(Y_{k}^{-1}-X_{k}\right) Y_{k} \geq 0
$$

and

$$
X_{k+1}-X_{k}=-A^{\star}\left(Y_{k+1}-Y_{k}\right) A \leq 0 .
$$

We have now proved (8) for $n=k+1$. Therefore, (8) is true for all $n$, and $\lim _{n \rightarrow \infty} X_{n}$ and $\lim _{n \rightarrow \infty} Y_{n}$ exist. So, we have $\lim X_{n}=X_{\infty}$ and $\lim Y_{n}=X_{\infty}^{-1}$.

Similar to Theorem 2, we can state the following theorem.

Theorem 4 If matrix equation (2) has a positive definite solution for Algorithm (7) and any $\epsilon>0$, then we have

$$
\left\|Y_{n+1}-X_{\infty}^{-1}\right\| \leq\left(\left\|A X_{\infty}^{-1}\right\|+\left\|B X_{\infty}^{-1}\right\|+\epsilon\right)^{2}\left\|Y_{n}-X_{\infty}^{-1}\right\|
$$

and

$$
\left\|X_{n}-X_{\infty}\right\| \leq\left(\|A\|^{2}+\|B\|^{2}\right)\left\|Y_{n}-X_{\infty}^{-1}\right\|
$$

for all $n$ large enough.

Now, we can see that Algorithm (7) can be faster than Algorithm (3) from the estimates in Theorems 2 and 4.

\section{Algorithm 3 Take}

$$
\left\{\begin{array}{l}
X_{0}=I, \quad Y_{0}=I, \\
Y_{n+1}=\left(I-X_{n}\right) Y_{n}+I, \\
X_{n+1}=I-A^{\star} Y_{n+1} A-B^{\star} Y_{n+1} B, \quad n=0,1,2, \ldots
\end{array}\right.
$$

Algorithm (10) requires only five matrix multiplications per step, whereas Algorithm (3) requires six matrix multiplications per step. 
Theorem 5 If matrix equation (2) has a positive definite solution and the two sequences $\left\{X_{n}\right\}$ and $\left\{Y_{n}\right\}$ are determined by Algorithm (10), then $\left\{X_{n}\right\}$ is monotone decreasing and converges to the maximal Hermitian positive definite solution $X_{\infty}$. Also, the sequence $\left\{Y_{n}\right\}$ defined in Algorithm (10) is a monotonically increasing sequence converging to $X_{\infty}^{-1}$.

Proof Let $X_{+}$be a positive definite solution of equation (2). We prove that

$$
X_{0} \geq X_{1} \geq \cdots \geq X_{n} \geq X_{+}
$$

and

$$
Y_{0} \leq Y_{1} \leq \cdots \leq Y_{n} \leq X_{+}^{-1}
$$

Since $X_{+}$is a solution of matrix equation (1), $X_{0}=I \geq X_{+}$. Also, we have $Y_{0}=Y_{1}=I$, and so

$$
X_{1}=I-A^{\star} A-B^{\star} B \geq I-A^{\star} X_{+}^{-1} A-B^{\star} X_{+}^{-1} B=X_{+}
$$

i.e., $X_{0} \geq X_{1} \geq X_{+}$.

For the sequence $\left\{Y_{n}\right\}$, since $I \leq X_{+}^{-1}, Y_{0}=Y_{1}=I \leq X_{+}^{-1}$.

Thus inequalities (11) and (12) are true for $n=1$. Now, assume that inequalities (11) and (12) are true for $n=k$, i.e.,

$$
X_{0} \geq X_{1} \geq \cdots \geq X_{k} \geq X_{+}
$$

and

$$
Y_{0} \leq Y_{1} \leq \cdots \leq Y_{k} \leq X_{+}^{-1}
$$

We show that inequalities (11) and (12) are true for $n=k+1$. We have

$$
Y_{k+1}=\left(I-X_{k}\right) Y_{k}+I \geq\left(I-X_{k-1}\right) Y_{k-1}+I=Y_{k}
$$

and

$$
Y_{k+1}=\left(I-X_{k}\right) Y_{k}+I \leq\left(I-X_{+}\right) X_{+}^{-1}+I=X_{+}^{-1},
$$

i.e., $Y_{k} \leq Y_{k+1} \leq X_{+}^{-1}$. Then

$$
X_{k}-X_{k+1}=A^{\star}\left(Y_{k+1}-Y_{k}\right) A+B^{\star}\left(Y_{k+1}-Y_{k}\right) B,
$$

and $X_{k+1} \leq X_{k}$, since $Y_{k} \leq Y_{k+1}$. On the other hand,

$$
\begin{aligned}
X_{k+1} & =I-A^{\star} Y_{k+1} A-B^{\star} Y_{k+1} B \\
& \geq I-A^{\star} X_{+}^{-1} A-B^{\star} X_{+}^{-1} B \\
& =X_{+}
\end{aligned}
$$

i.e., $X_{k} \geq X_{k+1} \geq X_{+}$. 
Then the above inequalities are true for all $n$, also $\lim _{n \rightarrow \infty} X_{n}$ and $\lim _{n \rightarrow \infty} Y_{n}$ exist. By taking limit on Algorithm (10), we have $\lim _{n \rightarrow \infty} X_{n}=X_{\infty}$ and $\lim _{n \rightarrow \infty} Y_{n}=X_{\infty}^{-1}$, where $X_{\infty}$ is the maximal positive definite solution of matrix equation (2).

By Algorithm (10), we have $I-X_{n} Y_{n}=Y_{n+1}-Y_{n}$. Then, for small $\epsilon>0,\left\|I-X_{n} Y_{n}\right\|$ can be one stopping condition.

Theorem 6 If matrix equation (2) has a positive definite solution and after $n$ iterative steps of Algorithm (10), the inequality $\left\|I-X_{n} Y_{n}\right\|<\epsilon$ implies

$$
\left\|X_{n}+A^{\star} X_{n}^{-1} A+B^{\star} X_{n}^{-1} B-I\right\| \leq \epsilon\left(\|A\|^{2}+\|B\|^{2}\right)\left\|X_{\infty}^{-1}\right\|
$$

Proof Since

$$
\begin{gathered}
X_{n}+A^{\star} X_{n}^{-1} A+B^{\star} X_{n}^{-1} B-I=X_{n}-X_{n+1}+A^{\star}\left(X_{n}^{-1}-Y_{n+1}\right) A+B^{\star}\left(X_{n}^{-1}-Y_{n+1}\right) B \\
=A^{\star}\left(Y_{n+1}-X_{n}^{-1}+X_{n}^{-1}-Y_{n}\right) A \\
+B^{\star}\left(Y_{n+1}-X_{n}^{-1}+X_{n}^{-1}-Y_{n}\right) B \\
+A^{\star}\left(X_{n}^{-1}-Y_{n+1}\right) A+B^{\star}\left(X_{n}^{-1}-Y_{n+1}\right) B \\
=A^{\star} X_{n}^{-1}\left(I-X_{n} Y_{n}\right) A+B^{\star} X_{n}^{-1}\left(I-X_{n} Y_{n}\right) B, \\
\left\|X_{n}+A^{\star} X_{n}^{-1} A+B^{\star} X_{n}^{-1} B-I\right\| \leq\left(\|A\|^{2}+\|B\|^{2}\right)\left\|X_{\infty}^{-1}\right\|\left\|I-X_{n} Y_{n}\right\| \\
\leq \epsilon\left(\|A\|^{2}+\|B\|^{2}\right)\left\|X_{\infty}^{-1}\right\| .
\end{gathered}
$$

This completes the proof.

Theorem 7 If $X_{n}>0$ for every $n$, then matrix equation(2) has a Hermitian positive definite solution.

Proof Since $X_{n}>0$ for every $n$, the proof of the monotonicity of $\left\{Y_{n}\right\}$ and $\left\{X_{n}\right\}$ noted in Theorem 5 remains valid. Therefore, the sequence $\left\{X_{n}\right\}$ is monotone decreasing and bounded from below by the zero matrix. Then $\lim _{n \rightarrow \infty} X_{n}=X$ exists. We claim that the sequence $\left\{Y_{n}\right\}$ is bounded above. Suppose that it does not hold. Then, for every $m>0$, there exists $n_{m}$ such that $m I<Y_{n_{m}}$. Since each $X_{n}$ is positive definite for every $n$, we have

$$
A^{\star} Y_{n} A+B^{\star} Y_{n} B<I \quad \text { for every } n \text {. }
$$

Furthermore, since $A$ or $B$ are nonsingular for every $m>0$, we have

$$
m\left(A^{\star} A+B^{\star} B\right)<A^{\star} Y_{n_{m}} A+B^{\star} Y_{n_{m}} B<I .
$$

By [16, Lemma 1.2],

$$
m\left(A^{\star} A+B^{\star} B\right)<I \quad \text { for every } m,
$$

which is a contradiction. Then the sequence $\left\{Y_{n}\right\}$ is bounded above and convergent. Suppose that $\lim _{n \rightarrow \infty} Y_{n}=Y$. As $Y_{0}=I$ and $\left\{Y_{n}\right\}$ is monotone increasing, $Y \leq I$. Taking limit 
in Algorithm (10) implies that

$$
\begin{aligned}
& Y=(I-X) Y+I, \\
& X=I-A^{\star} Y A-B^{\star} Y B .
\end{aligned}
$$

Since $Y \leq I, X=Y^{-1}>0$, and hence $X=I-A^{\star} X^{-1} A-B^{\star} X^{-1} B$. Then matrix equation (2) has a positive definite solution.

Theorem 8 If matrix equation (2) has a positive definite solution and $\|A\|<\frac{1}{2}$ and $\|B\|<$ $\frac{1}{2}$, then the sequence $\left\{X_{n}\right\}$ defined in Algorithm (10) satisfies

$$
\left\|Y_{n+1}-X_{\infty}^{-1}\right\| \leq\left(\left\|A X_{\infty}^{-1}\right\|+\left\|B X_{\infty}^{-1}\right\|\right)\left\|Y_{n}-X_{\infty}^{-1}\right\|
$$

and

$$
\left\|X_{n+1}-X_{\infty}\right\| \leq\left(\|A\|^{2}+\|B\|^{2}\right)\left\|Y_{n}-X_{\infty}^{-1}\right\|
$$

for all $n$ large enough.

Proof From Algorithm (10), we have

$$
\begin{aligned}
Y_{n+1} & =\left(I-X_{n}\right) Y_{n}+I \\
& =A^{\star} Y_{n} A Y_{n}+B^{\star} Y_{n} B Y_{n}+I \\
& =A^{\star}\left(Y_{n}+X_{\infty}^{-1}-X_{\infty}^{-1}\right) A Y_{n}+B^{\star}\left(Y_{n}+X_{\infty}^{-1}-X_{\infty}^{-1}\right) B Y_{n}+I \\
& =A^{\star}\left(Y_{n}-X_{\infty}^{-1}\right) A Y_{n}+B^{\star}\left(Y_{n}-X_{\infty}^{-1}\right) B Y_{n}+A^{\star} X_{\infty}^{-1} A Y_{n}+B^{\star} X_{\infty}^{-1} B Y_{n}+Y_{n}-Y_{n}+I \\
& =A^{\star}\left(Y_{n}-X_{\infty}^{-1}\right) A Y_{n}+B^{\star}\left(Y_{n}-X_{\infty}^{-1}\right) B Y_{n}-\left(I-A^{\star} X_{\infty}^{-1} A-B^{\star} X_{\infty}^{-1} B\right) Y_{n}+Y_{n}+I \\
& =A^{\star}\left(Y_{n}-X_{\infty}^{-1}\right) A Y_{n}+B^{\star}\left(Y_{n}-X_{\infty}^{-1}\right) B Y_{n}-X_{\infty} Y_{n}+Y_{n}+I .
\end{aligned}
$$

Thus

$$
\begin{aligned}
X_{\infty}^{-1}-Y_{n+1}= & X_{\infty}^{-1}+A^{\star}\left(X_{\infty}^{-1}-Y_{n}\right) A Y_{n}+B^{\star}\left(X_{\infty}^{-1}-Y_{n}\right) B Y_{n}+X_{\infty} Y_{n}-Y_{n}-I \\
= & A^{\star}\left(X_{\infty}^{-1}-Y_{n}\right) A Y_{n}+B^{\star}\left(X_{\infty}^{-1}-Y_{n}\right) B Y_{n}+\left(I-X_{\infty}\right)\left(X_{\infty}^{-1}-Y_{n}\right) \\
= & A^{\star}\left(X_{\infty}^{-1}-Y_{n}\right) A Y_{n}+B^{\star}\left(X_{\infty}^{-1}-Y_{n}\right) B Y_{n} \\
& +A^{\star} X_{\infty}^{-1} A\left(X_{\infty}^{-1}-Y_{n}\right)+B^{\star} X_{\infty}^{-1} B\left(X_{\infty}^{-1}-Y_{n}\right) .
\end{aligned}
$$

Therefore, we have

$$
\begin{aligned}
\left\|X_{\infty}^{-1}-Y_{n+1}\right\| & \leq\left(\left\|A^{\star}\right\|\left\|A Y_{n}\right\|+\left\|B^{\star}\right\|\left\|B Y_{n}\right\|+\left\|A^{\star} X_{\infty}^{-1} A\right\|+\left\|B^{\star} X_{\infty}^{-1} B\right\|\right)\left\|X_{\infty}^{-1}-Y_{n}\right\| \\
& \leq\left(\left(\left\|A Y_{n}\right\|+\left\|X_{\infty}^{-1} A\right\|\right)\left\|A^{\star}\right\|+\left(\left\|B Y_{n}\right\|+\left\|X_{\infty}^{-1} B\right\|\right)\left\|B^{\star}\right\|\right)\left\|X_{\infty}^{-1}-Y_{n}\right\| .
\end{aligned}
$$

Now, since $\lim _{n \rightarrow \infty} Y_{n}=X_{\infty}^{-1}$, inequality (15) follows. Also, inequality (16) is true since

$$
X_{n+1}-X_{\infty}=A^{\star}\left(X_{\infty}^{-1}-Y_{n}\right) A+B^{\star}\left(X_{\infty}^{-1}-Y_{n}\right) B
$$

This completes the proof. 


\section{Numerical examples}

In this section, we present some numerical examples to show the effectiveness of the new inversion-free variant of the basic fixed point iteration methods. Hermitian positive definite solutions of matrix equation (2) for different matrices $A$ and $B$ are computed. We will compare the suggested algorithms, Algorithm (7) and Algorithm (10), by Algorithm (3). All programs were written in MATLAB.

Example 1 Consider equation (2) with

$$
A=\frac{1}{200}\left(\begin{array}{ccc}
0.2 & -0.1 & 0.3 \\
0.56 & 0.3 & -0.7 \\
0.2 & 0.5 & 0.6
\end{array}\right), \quad B=\frac{1}{20}\left(\begin{array}{ccc}
0.46 & -0.01 & 0.020 \\
-0.15 & -0.488 & -0.060 \\
0.04 & -0.01 & -0.120
\end{array}\right)
$$

Algorithm (3) needs six iterations to get the solution

$$
\begin{aligned}
& X=\left(\begin{array}{ccc}
0.999400612248657 & -0.000176704506272 & -0.000028208026789 \\
-0.000176704506272 & 0.999395021004650 & -0.000077249011425 \\
-0.000028208026789 & -0.000077249011425 & 0.999930483901903
\end{array}\right), \\
& \left\|X+A^{\star} X^{-1} A+B^{\star} X^{-1} B-I\right\|_{\infty}=1.3885 \mathrm{e}-013
\end{aligned}
$$

Algorithm (7) needs six iterations to get the solution

$$
\begin{aligned}
& X=\left(\begin{array}{ccc}
0.999400612248567 & -0.000176704506276 & -0.000028208026792 \\
-0.000176704506276 & 0.999395021004514 & -0.000077249011443 \\
-0.000028208026792 & -0.000077249011443 & 0.999930483901898
\end{array}\right), \\
& \left\|X+A^{\star} X^{-1} A+B^{\star} X^{-1} B-I\right\|_{\infty}=6.7763 \mathrm{e}-021 .
\end{aligned}
$$

We can easily see that Algorithm (7) is more accurate than Algorithm (3).

Algorithm (10) needs six iterations to get the solution

$$
\begin{aligned}
& X=\left(\begin{array}{ccc}
0.999400612248567 & -0.000176704506276 & -0.000028208026792 \\
-0.000176704506276 & 0.999395021004514 & -0.000077249011443 \\
-0.000028208026792 & -0.000077249011443 & 0.999930483901898
\end{array}\right), \\
& \left\|X+A^{\star} X^{-1} A+B^{\star} X^{-1} B-I\right\|_{\infty}=6.7763 \mathrm{e}-021 .
\end{aligned}
$$

Example 2 Consider equation (2) with

$$
A=\frac{1}{820}\left(\begin{array}{lllll}
41 & 15 & 23 & 35 & 66 \\
25 & 12 & 27 & 45 & 21 \\
23 & 27 & 28 & 16 & 24 \\
15 & 45 & 16 & 52 & 65 \\
66 & 21 & 24 & 65 & 35
\end{array}\right), \quad B=\frac{1}{830}\left(\begin{array}{ccccc}
23 & 21 & 23 & 25 & 32 \\
21 & 45 & 60 & 42 & 33 \\
23 & 24 & 34 & 18 & 17 \\
13 & 42 & 18 & 44 & 30 \\
32 & 33 & 26 & 30 & 26
\end{array}\right)
$$


Algorithm (3) after 21 iterations gives the solution

$$
\begin{gathered}
X=\left(\begin{array}{ccc}
0.98393799066 & -0.01161748103 & -0.01233926321 \\
-0.01161748103 & 0.98497686219 & -0.01315828865 \\
-0.01233926321 & -0.01315828865 & 0.98561286596 \\
-0.01833845539 & -0.01745583944 & -0.01623773649 \\
-0.01633619168 & -0.01639741581 & -0.01467582916 \\
\left\|X+A^{\star} X^{-1} A+B^{\star} X^{-1} B-I\right\|_{\infty}=3.7975 \mathrm{e}-013
\end{array}\right.
\end{gathered}
$$

$-0.01833845539$

$-0.01745583944$

$-0.01623773649$

0.97439947749

$-0.02237728728$

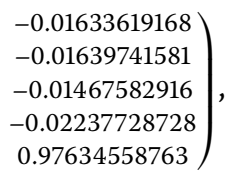

Algorithm (7) after 21 iterations gives the solution

$$
\begin{aligned}
& X=\left(\begin{array}{ccccc}
0.98393799066 & -0.01161748103 & -0.01233926321 & -0.01833845539 & -0.01633619168 \\
-0.01161748103 & 0.98497686219 & -0.01315828865 & -0.01745583944 & -0.01639741581 \\
-0.01233926321 & -0.01315828865 & 0.98561286596 & -0.01623773649 & -0.01467582916 \\
-0.01833845539 & -0.01745583944 & -0.01623773649 & 0.97439947749 & -0.02237728728 \\
-0.01633619168 & -0.01639741581 & -0.01467582916 & -0.02237728728 & 0.97634558763
\end{array}\right), \\
& \left\|X+A^{\star} X^{-1} A+B^{\star} X^{-1} B-I\right\|_{\infty}=6.0963 \mathrm{e}-018 .
\end{aligned}
$$

Algorithm (10) after 21 iterations gives the solution

$$
X=\left(\begin{array}{ccccc}
0.98393799066 & -0.01161748103 & -0.01233926321 & -0.01833845539 & -0.01633619168 \\
-0.01161748103 & 0.98497686219 & -0.01315828865 & -0.01745583944 & -0.01639741581 \\
-0.01233926321 & -0.01315828865 & 0.98561286596 & -0.01623773649 & -0.01467582916 \\
-0.01833845539 & -0.01745583944 & -0.01623773649 & 0.97439947749 & -0.02237728728 \\
-0.01633619168 & -0.01639741581 & -0.01467582916 & -0.02237728728 & 0.97634558763
\end{array}\right) \text {, }
$$

$$
\left\|X+A^{\star} X^{-1} A+B^{\star} X^{-1} B-I\right\|_{\infty}=6.1220 \mathrm{e}-018 .
$$

We can see that Algorithm (10) needs to find a Hermitian positive definite solution.

\section{Competing interests}

The authors declare that they have no competing interests.

\section{Authors' contributions}

All authors carried out the proof. All authors conceived of the study and participated in its design and coordination. All authors read and approved the final manuscript.

\section{Author details}

${ }^{1}$ Department of Mathematics and Computer Science, Amirkabir University of Technology, Hafez Ave., P.O. Box 15914, Tehran, Iran. ${ }^{2}$ Department of Mathematics, Iran University of Science and Technology, Tehran, Iran. ${ }^{3}$ Research Institute for Natural Sciences, Hanyang University, Seoul, 133-791, Korea.

\section{Acknowledgements}

The authors are grateful to the two anonymous reviewers for their valuable comments and suggestions.

Received: 13 June 2013 Accepted: 22 July 2013 Published: 6 August 2013

\section{References}

1. Buzbee, BL, Golub, GH, Nielson, CW: On direct methods for solving Poisson's equations. SIAM J. Numer. Anal. 7, 627-656 (1970)

2. Housholder, AS: The Theory of Matrices in Numerical Analysis. Blaisdell, New York (1964)

3. Engwerda, JC, Ran, AC, Rijkeboer, AL: Necessary and sufficient conditions for the existence of a positive definite solution of the matrix equation $X+A^{\star} X^{-1} A=Q$. Linear Algebra Appl. 186, 255-275 (1993)

4. Engwerda, JC: On the existence of a positive definite solution of the matrix equation $X+A^{\top} X^{-1} A=I$. Linear Algebra Appl. 194, 91-108 (1993)

5. Lancaster, P, Rodman, L: Algebraic Riccati Equations. Oxford Science Publishers, Oxford (1995)

6. Zhan, $X$, Xie, J: On the matrix equation $X+A^{\top} X^{-1} A=1$. Linear Algebra Appl. 247, 337-345 (1996)

7. Anderson, WN, Morley, TD, Trapp, GE: Positive solutions of the matrix equation $X=A-B^{\star} X^{-1} B$. Linear Algebra Appl. 134, 53-62 (1990)

8. Zhan, X: Computing the extremal positive definite solutions of a matrix equation. SIAM J. Sci. Comput. 17, 1167-1174 (1996) 
9. Shufang, $X$ : On the maximal solution for the matrix equation $X+A^{\top} X^{-1} A=1$. Acta Sci. Nat. Univ. Pekin. 36, 29-38 (2000)

10. Guo, $\mathrm{CH}$, Lancaster, P: Iterative solution of two matrix equations. Math. Comput. 68, 1589-1603 (1999)

11. Meini, $B$ : Efficient computation of the extreme solutions of $X+A^{\star} X^{-1} A=Q$ and $X-A^{\star} X^{-1} A=Q$. Math. Comput. 71, 1189-1204 (2002)

12. El-Sayed, SM, Ran, ACM: On an iteration method for solving a class of nonlinear matrix equation. SIAM J. Matrix Anal. Appl. 23, 632-645 (2001)

13. Ivanov, IG, Hasanov, VI, Uhlig, F: Improved methods and starting values to solve the matrix equations $X \pm A^{\star} X^{-1} A=I$ iteratively. Math. Comput. 74, 263-278 (2004)

14. El-Sayed, SM, Al-Dbiban, AM: A new inversion free iteration for solving the equation $X+A^{\star} X^{-1} A=Q$. J. Comput. Appl. Math. 181, 148-156 (2005)

15. Long, $\mathrm{JH}, \mathrm{Hu}, \mathrm{XY}$, Zhang, L: On the Hermitian positive definite solution of the matrix equation $X+A^{\star} X^{-1} A+B^{\star} X^{-1} B=1$. Bull. Braz. Math. Soc. 39, 371-386 (2008)

16. Chen, G, Huang, X, Yang, X: Vector Optimization: Set-Valued and Variational Analysis. Springer, Berlin (2005)

doi:10.1186/1687-1847-2013-229

Cite this article as: Vaezzadeh et al.: The iterative methods for solving nonlinear matrix equation

$X+A^{\star} X^{-1} A+B^{\star} X^{-1} B=Q$. Advances in Difference Equations 2013 2013:229.

\section{Submit your manuscript to a SpringerOpen ${ }^{\circ}$ journal and benefit from:}

- Convenient online submission

- Rigorous peer review

- Immediate publication on acceptance

- Open access: articles freely available online

- High visibility within the field

- Retaining the copyright to your article 